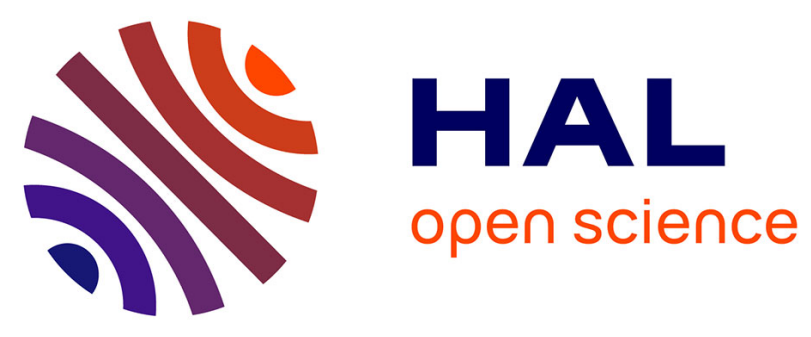

\title{
Modulation transfer function measurement of a multichannel optical system
}

Florence de La Barrière, Guillaume Druart, Nicolas Guérineau, Jean Albert Taboury, Jérôme Primot, Joël Deschamps

\section{- To cite this version:}

Florence de La Barrière, Guillaume Druart, Nicolas Guérineau, Jean Albert Taboury, Jérôme Primot, et al.. Modulation transfer function measurement of a multichannel optical system. Applied optics, 2010, 49 (15), pp.2879-2890. 10.1364/AO.49.002879 . hal-00569985

\section{HAL Id: hal-00569985 \\ https://hal-iogs.archives-ouvertes.fr/hal-00569985}

Submitted on 25 May 2012

HAL is a multi-disciplinary open access archive for the deposit and dissemination of scientific research documents, whether they are published or not. The documents may come from teaching and research institutions in France or abroad, or from public or private research centers.
L'archive ouverte pluridisciplinaire HAL, est destinée au dépôt et à la diffusion de documents scientifiques de niveau recherche, publiés ou non, émanant des établissements d'enseignement et de recherche français ou étrangers, des laboratoires publics ou privés. 


\title{
Modulation transfer function measurement of a multichannel optical system
}

\author{
Florence de la Barrière, ${ }^{1, \star}$ Guillaume Druart, ${ }^{1}$ Nicolas Guérineau, ${ }^{1}$ Jean Taboury, ${ }^{2}$ \\ Jérôme Primot, ${ }^{1}$ and Joël Deschamps ${ }^{1}$ \\ ${ }^{1}$ ONERA, Chemin de la Hunière, 91761 Palaiseau Cedex, France \\ ${ }^{2}$ Institut d'Optique, Campus Polytechnique RD 128, 91127 Palaiseau Cedex, France \\ ${ }^{\star}$ Corresponding author: florence.de_la_barriere@onera.fr
}

Received 28 January 2010; revised 8 April 2010; accepted 9 April 2010; posted 23 April 2010 (Doc. ID 123424); published 14 May 2010

\begin{abstract}
We present a new method to measure the modulation transfer function (MTF) beyond the Nyquist frequency of a multichannel imaging system for which all the channels have parallel optical axes. Such a multichannel optical system produces a set of undersampled subimages. If the subimages contain nonredundant information, high spatial frequencies are folded between low spatial frequencies, leading to the possible extraction of frequencies higher than the Nyquist frequency. The measurement of the MTF of the multichannel system leads to the estimation of the resolution enhancement of the final image that can be obtained by applying a postprocessing algorithm to the collection of undersampled subimages. Experimental images are presented to validate this method. (C) 2010 Optical Society of America
\end{abstract}

OCIS codes: $\quad 110.0110,110.4100,110.3080,110.4190,110.4155,130.3990$.

\section{Introduction}

Today, both military and civilian applications require miniaturized and cheap optical systems. The constraints on the size and weight of miniaturized systems are so demanding that downscaled versions of traditional optical systems with a single optical axis are reaching their limits. Indeed, classical optics cannot be downscaled without a loss of resolved points [1]. A solution to overcome this limitation can be found by looking at nature, where small invertebrates have developed compound eyes [2]. There are two types of compound eye: apposition compound eyes and superposition compound eyes. For both architectures, the main idea is to divide the information of the whole scene into several optical channels. Various compact architectures inspired by insects' visual systems have already been proposed [3-10], and they differ in the way of dividing the information contained in the whole scene. The scene can be spatially split into different optical channels: each channel

0003-6935/10/152879-12\$15.00/0

(C) 2010 Optical Society of America images a small part of the scene, resulting in a large field of view (FOV) system. This effect is obtained by tilting the optical axis of each channel differently [5-7]. The sampling of the scene can also be split into the different channels. This way of dividing the information is presented in the concept TOMBO (thin observation module by bound optics) $[8,9]$ : the optical system collects nonredundant and undersampled images of the same scene, the nonredundancy being usually obtained with subpixel shifts between the optical channels. The information contained in the scene can also be spectrally distributed in the different optical channels. A multispectral TOMBO system is proposed in [10]: an interference filter array is placed in front of each optical channel, so that the optical system images the same scene through different spectral bands. Then an image processing method based on the multispectral images provides spectral information of the scene.

For multichannel systems based on the TOMBO principle, an important issue is to retrieve a single image with high resolution from the collection of undersampled low-resolution ones. This is achieved by applying superresolution image processing [11-15]. 
The design of multichannel optical systems and the image processing method have to be jointly optimized in order to fully benefit from the resolution enhancement provided by superresolution: this approach is known as codesign $[16,17]$. Some papers have already proposed superresolution performance models based on optical characteristics of the camera used in the experiments [18-20]: the merit function defined in [18] takes into account the FOV, the angular resolution, the sensitivity, and the volume of the optical system in a single equation. In [19], the performance of several superresolution algorithms is evaluated by a merit function based on the sensor transfer function, the number of frames, and the interframe translation motion. In this example, as in [20], where the shifts between the subimages are determined once and for all by calibrating the camera, the knowledge of the modulation transfer function (MTF) of the multichannel system would greatly improve the final image resolution enhancement by taking into account the aberrations of the optical system. Therefore, the experimental evaluation of the optical quality of multichannel imaging systems is a crucial issue.

Section 2 summarizes the principles linked to optical miniaturization, which lead to designing multichannel systems based on the TOMBO principle. We show that such systems are theoretically equivalent to single-channel imaging systems correctly sampled by a scanning monodetector. Based on this model, we define the global MTF of a multichannel system that can be used as a new figure of merit to evaluate the performance of the system in terms of resolution enhancement of the final image. In Section $\underline{3}$, relying on previous work on MTF measurement, we propose a new and simple method to measure this global MTF beyond the Nyquist frequency; it requires only a point source and no movable parts. In the case of an optical system for which no subpixel shift is introduced between the optical channels, the nonredundancy can be obtained by tilting the optical system in relation to the axes of the detector. Section $\underline{4}$ illustrates this method with experimental data acquired by a multichannel camera working in the $[3 \mu \mathrm{m} ; 5 \mu \mathrm{m}]$ spectral range, called MULTICAM [21]. Although the method is illustrated in the infrared domain, it can be used for any multichannel optical system, whatever its spectral range. The measurement of frequencies greater than the Nyquist frequency suggests that a resolution enhancement on the final image can be expected with a postprocessing algorithm: we will give an illustration of this resolution enhancement with an experimental image acquired by this camera.

\section{Principles for Optical Miniaturization Leading to the Design of Multichannel Systems}

In this section, we first define useful notions to describe the quality of an optical system, and we rely on these definitions to evaluate the impact of miniaturizing an optical system on its image quality. Then, we recall a method used to miniaturize an optical system while maintaining the same image resolution; this method is based on the design of a multichannel system according to the TOMBO principle [8]. Then, we show that a multichannel optical system associated with a focal plane array is equivalent, in terms of sampling of the scene, to a single-channel optical system associated with a scanning monodetector. These considerations lead to defining a global MTF for a multichannel system in order to evaluate its optical performance.

\section{A. Definitions and Notation}

\section{MTF of an Optical System}

The quality of an optical system can be described by its optical transfer function (OTF), which is the Fourier transform of the point spread function (PSF). The transfer function of the pixel $\left(\mathrm{TF}_{\text {pixel }}\right)$ takes into account the finite size of the pixel $t_{\mathrm{pix}}$. The cutoff frequency of the pixel is defined as $1 / t_{\mathrm{pix}}$ and corresponds to the first zero of the pixel transfer function. The moduli of the OTF and of $\mathrm{TF}_{\text {pixel }}$ are illustrated in Fig. 1. The transfer function of the whole system is the product of the OTF times $\mathrm{TF}_{\text {pixel }}$; its modulus is the MTF:

$$
\mathrm{MTF}=\left|\mathrm{OTF} \times \mathrm{TF}_{\text {pixel }}\right|
$$

\section{Image Sampling and Aliasing}

Due to sampling on the detector, replications of the MTF appear around the multiples of the sampling frequency $f_{s}=1 / p_{s}$, where $p_{s}$ is the sampling pitch of the detector. If the MTF cutoff frequency is greater than the Nyquist frequency $f_{\mathrm{Ny}}$ (which is defined as half the sampling frequency, $f_{\mathrm{Ny}}=f_{s} / 2$ ), high frequencies (greater than $f_{\mathrm{Ny}}$ ) are folded on low frequencies (lower than $f_{\mathrm{Ny}}$ ), which are thus deteriorated. This phenomenon is known as aliasing and is illustrated in Fig. 2.

\section{Angular Resolution}

The maximum resolvable spatial frequency $\nu_{\max }$ of the system is the ability to distinguish small details, which we define by the following equation:

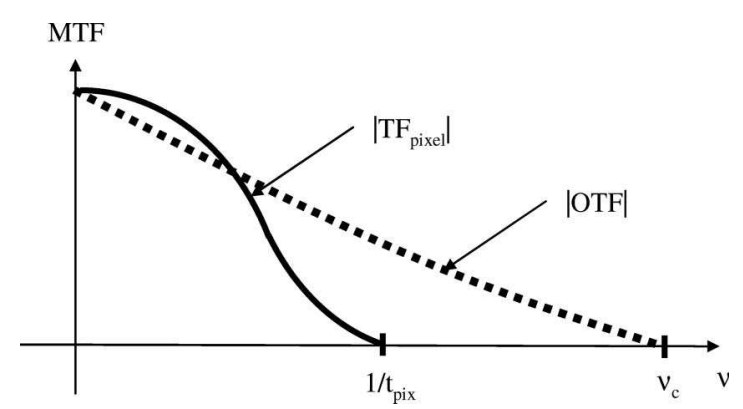

Fig. 1. Moduli of the pixel transfer function $\left(\left|\mathrm{TF}_{\text {pixel }}\right|\right)$ and of the optical transfer function $(|\mathrm{OTF}|)$. 


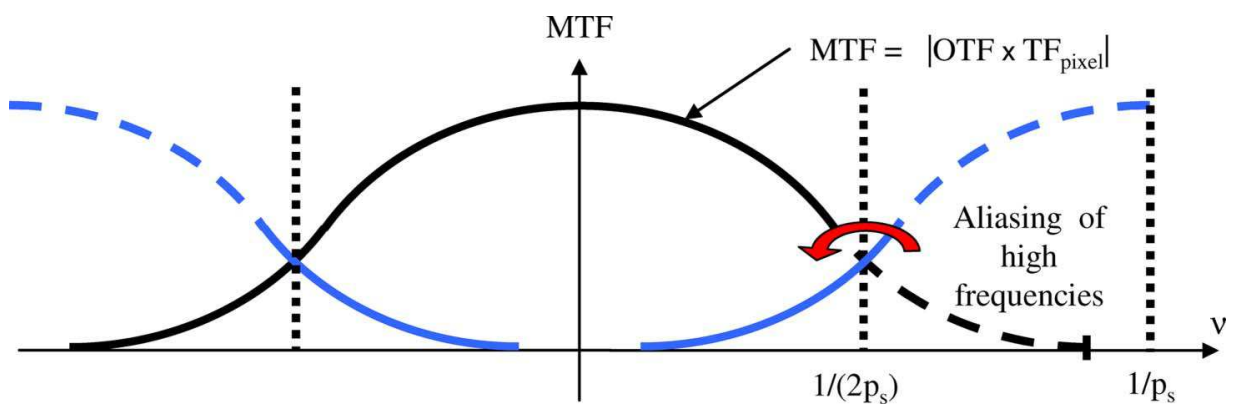

Fig. 2. (Color online) Aliasing on the MTF of an optical system.

$$
\nu_{\max }=\min \left(1 /\left(2 p_{s}\right), \nu_{c}, 1 / t_{\mathrm{pix}}\right)
$$

where $\nu_{c}$ is the cutoff frequency of the optical system (the frequency for which the OTF is equal to 0 ). For a diffraction-limited optical system with an $f$-number \#, working at wavelength $\lambda$ for an object at infinity, $\nu_{c}$ is equal to $1 /(\lambda \#)$.

The angular resolution (IFOV) of the system is linked to its maximum resolvable spatial frequency $\nu_{\max }$; we define IFOV by the following equation:

$$
\mathrm{IFOV}=\frac{1}{f \nu_{\max }}
$$

where $f$ is the focal length of the optical system. IFOV is either limited by the Nyquist frequency linked to the detector (if $\nu_{\max }=1 /\left(2 p_{s}\right)$ ) or by filtering effects (linked to the optical system if $\nu_{\max }=\nu_{c}$ or to the pixel if $\nu_{\max }=1 / t_{\mathrm{pix}}$ ).

B. Miniaturization of an Optical System while Maintaining a Constant Angular Resolution

The miniaturization of an optical system can be performed by reducing its focal length $f$ while maintaining the same FOV. This means that the focal length $f$ and the diameter $\Phi$ of a classical optical system have to be downscaled simultaneously by the same factor $M$. Thus, the image surface is reduced by a factor $M^{2}$, as illustrated in Fig. 3(a).

In most cases, classical optical systems are designed so that their resolution is limited by the sampling due to the detector, which means that the Nyquist frequency is lower than the OTF cutoff frequency, leading to aliasing effects. In this case, Eq. ( $\underline{3})$ becomes

$$
\mathrm{IFOV}=\frac{1}{f \times f_{\mathrm{Ny}}}=\frac{2 p_{s}}{f}
$$

We can see from Eq. (4) that if we want to downscale the focal length of the optical system by a factor $M$ while maintaining a constant IFOV, we have to reduce the sampling pitch, and as a consequence, the size of the pixel, by a factor $M$ also. However, the reduction of the pixel pitch reaches technological limits because of cross-talk between adjacent pixels [22]. If the pixel pitch remains the same, the number of resolved points in the image decreases [1], as shown in Fig. 3(b).

Equation (3) shows that another solution to downscale the focal length by a factor $M$ while maintaining a constant IFOV is to increase the maximum

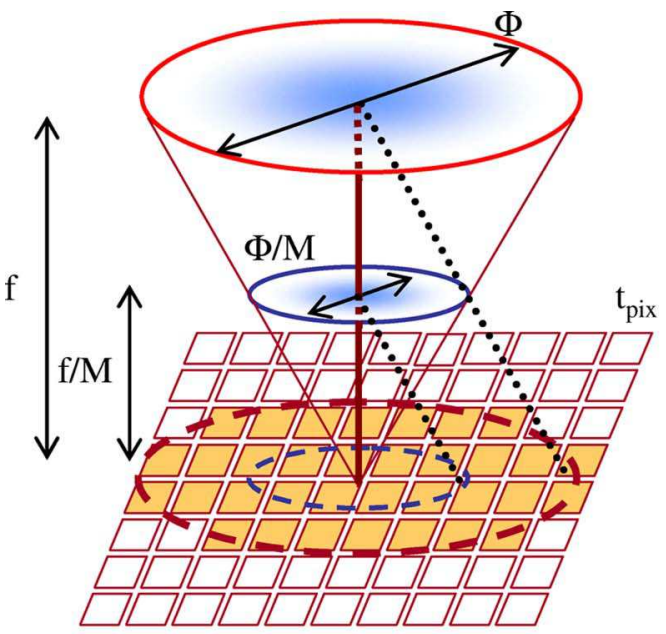

(a)
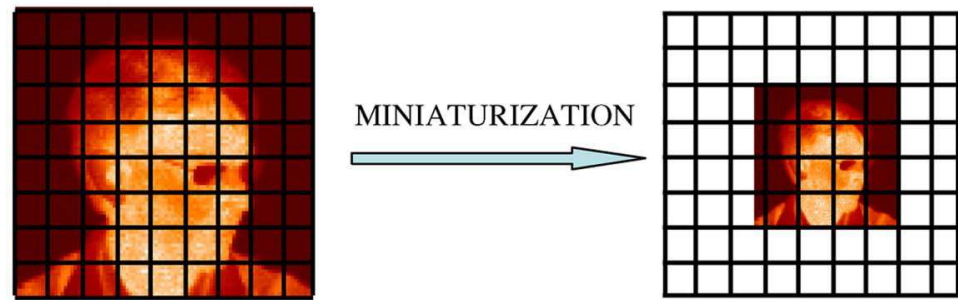

Fig. 3. (Color online) (a) Miniaturization of an optical system by downscaling its focal length $f$ and its diameter $\Phi$ by the same factor $M$. (b) Reduction of the number of resolved points in the image while maintaining the same pixel pitch on the detector. 
resolvable spatial frequency $\nu_{\max }$ by a factor $M$. This can be achieved if $\nu_{\max }$ is limited by the pixel cutoff frequency $1 / t_{\text {pix }}$ rather than the Nyquist frequency related to the detector sampling pitch [see Eq. (2)]. In this case, the cutoff frequency of the optical system $\nu_{c}$ has to be higher than the pixel cutoff frequency (which implies lowering the $f$-number \# of the optical system). Then, we have to propose a method to unfold frequencies higher than the Nyquist frequency. This can be achieved by designing a multichannel optical system based on the TOMBO principle [8], which is obtained by replicating a miniaturized system [see Fig. 4(a)].

C. Interest of Multichannel Optical Systems in Terms of Sampling of the Scene

By using the whole surface of the focal plane array, the multichannel system provides a set of undersampled subimages (see Fig. 4(b)), which contain nonredundant information. For the TOMBO system, the nonredundancy is achieved by choosing a period of the microlens array that is not a multiple of the pixel pitch of the detector, resulting in subpixel shifts between the subimages. This nonredundancy permits the retrieval of a single image with a high angular resolution from the collection of low-resolution subimages. The reconstruction of a high-resolution image is performed by applying a superresolution algorithm whose principle is based on the sampling theorem of Papoulis [23]: the latter says that a band-limited signal can be completely retrieved from $M$ nonredundant signals that are undersampled by a factor $1 / M$. In other words, the nonredundancy between the subimages splits the sampling of the scene between the different optical channels.

According to the Shannon theorem, if the maximum spatial frequency contained in the scene is equal to $1 / t_{\text {pix }}$, the sampling pitch $p_{s}$ has to fulfil the following condition in order to provide a correct sampling of the scene:

$$
p_{s} \leq t_{\mathrm{pix}} / 2 \text {. }
$$

This condition can be achieved by using a monodetector of size $t_{\text {pix }}$ that is scanned with a pitch equal to $t_{\text {pix }} / 2$. However, it cannot be achieved by using a focal plane array, for which $t_{\mathrm{pix}} \leq p_{s}$. If the fill factor of the pixels is equal to $1, p_{s}=t_{\mathrm{pix}}$; according to the TOMBO principle, if the sampling of the scene is split into different optical channels, the sampling pitch can be artificially reduced to $t_{\mathrm{pix}} / 2$ if we choose a reduction factor $M$ equal to 2 . Therefore, with a system based on the TOMBO principle, we can expect to fulfil Shannon's criterion and to retrieve frequencies up to twice the Nyquist frequency if the fill factor of the pixels is equal to 1 . Thus, a multichannel optical system used with a focal plane array combines the advantages of both a scanning monodetector (which samples the scene with a pitch equal to $t_{\text {pix }} / 2$ ) and a focal plane array (which provides an image of the scene in a single acquisition).

\section{Definition of the Global MTF of a Multichannel Imaging System}

In the previous paragraphs, we have seen that a multichannel system, associated with a focal plane array and combined with a superresolution algorithm, is theoretically equivalent to a single-channel imaging system that is $M$ times bigger and that is sampled by a monodetector with a sampling pitch equal to $t_{\text {pix }} / 2$. This observation suggests defining a global MTF of the multichannel optical system, which is different from the MTF of each channel separately.

Assuming that all the channels have the same optical quality, and that all the pixels of the focal plane array have the same spatial response $\mathrm{PSF}_{\text {pixel }}$, the PSF of a multichannel optical system can be described by the following equation:

$$
\begin{aligned}
\operatorname{PSF}_{m}(x, y)= & {\left[\operatorname{PSF}_{e}(x, y) * \operatorname{PSF}_{\text {pixel }}(x, y)\right] } \\
& *\left[\amalg_{d, d}(x, y) \times \operatorname{rect}\left(\frac{x}{a}\right) \times \operatorname{rect}\left(\frac{y}{b}\right)\right],
\end{aligned}
$$

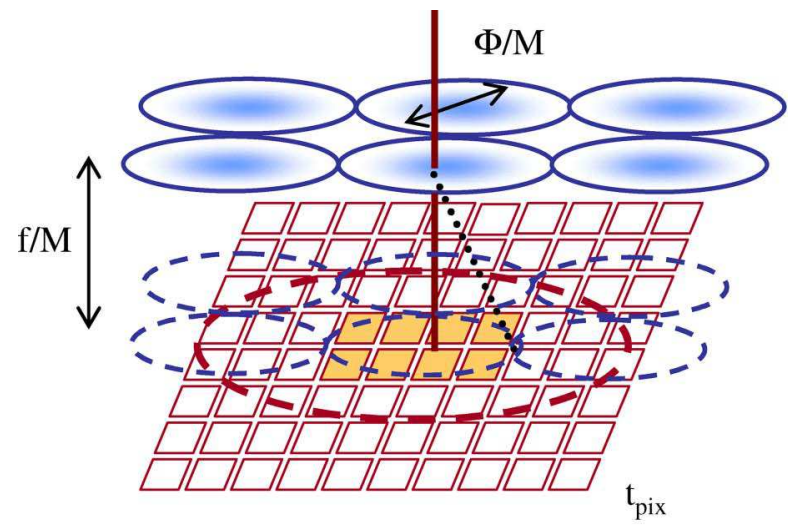

(a)

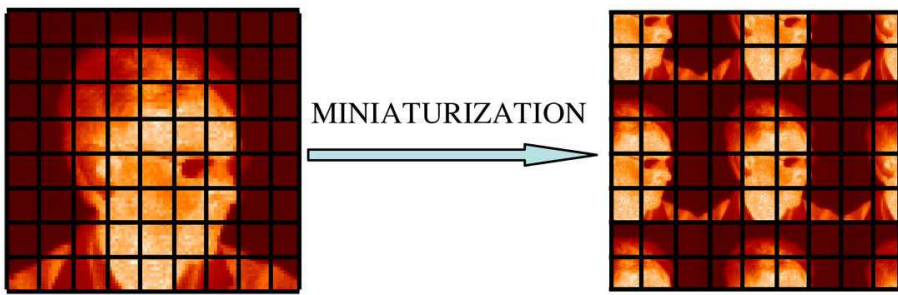

(b)

Fig. 4. (Color online) (a) Replication of a miniaturized imaging system, resulting in a multichannel optical system based on the TOMBO principle. (b) Image obtained on the detector surface. 
where $\operatorname{PSF}_{m}(x, y)$ is the PSF of the multichannel system, $\operatorname{PSF}_{e}(x, y)$ is the PSF of a single-channel optical system (if the elementary channels are limited by the diffraction, $\operatorname{PSF}_{e}(x, y)$ is an Airy pattern), $\amalg_{d, d}$ is a two-dimensional Dirac comb of period $d$ along the two axes $x$ and $y, d$ being the distance between two adjacent channels. This two-dimensional Dirac comb is limited by a rectangular aperture of dimensions $a$ and $b$ in the $x$ and $y$ directions, respectively, standing for the finite dimensions of the focal plane array. Thus, this aperture can be described mathematically by the expression $\operatorname{rect}(x / a) \times \operatorname{rect}(y / b)$, where $\operatorname{rect}(x)=1$ if $x \in[-1 / 2,1 / 2]$ and $\operatorname{rect}(x)=0$ otherwise.

The global MTF of the multichannel optical system is equal to the modulus of the Fourier transform of its PSF:

$$
\begin{aligned}
\operatorname{MTF}_{m}\left(\nu_{x}, \nu_{y}\right)= & \mid\left[\operatorname{OTF}_{e}\left(\nu_{x}, \nu_{y}\right) \times \operatorname{TF}_{\text {pixel }}\left(\nu_{x}, \nu_{y}\right)\right] \\
& \times\left[\amalg_{1 / d, 1 / d}\left(\nu_{x}, \nu_{y}\right)\right. \\
& \left.*\left(\operatorname{sinc}\left(a \nu_{x}\right) \times \operatorname{sinc}\left(b \nu_{y}\right)\right)\right] \mid,
\end{aligned}
$$

where $\operatorname{OTF}_{e}\left(\nu_{x}, \nu_{y}\right)$ is the OTF of a single-channel optical system, $\nu_{x}$ and $\nu_{y}$ being the variables in the Fourier domain, and sinc is a function defined by $\operatorname{sinc}(x)=1$ if $x=0$ and $\operatorname{sinc}(x)=\sin (\pi x) / \pi x$ otherwise.

The concept of global MTF for a multichannel optical system can be used as a new figure of merit to compare the optical performance of a multichannel system to an equivalent single-channel system. Knowing the MTF of a multichannel optical system provides several advantages. First, we can directly measure the resolution enhancement in the final image that could be obtained by applying a superresolution algorithm. It also gives the contrast of high frequencies beyond the Nyquist frequency. Moreover, the last step of the image processing is often to eliminate the blur caused by filtering effects. Knowing the MTF of a multichannel system enables to perform a deconvolution not only by the pixel transfer function, but also by the MTF of the optical system; in this way, we take into account the aberrations of the optical system to enhance the contrast of the final image.

Thus, an important issue is to measure experimentally the MTF of a multichannnel system.

\section{Description of a Simple Method to Measure the MTF of a Multichannel Imaging System}

\section{A. Overview of Previous Work on MTF Measurement}

Local and global methods have already been proposed to measure the MTF of optical systems and of focal plane arrays (FPAs). Local methods evaluate the MTF on a restrictive zone of the FPA, or even for a single pixel. They involve a movable knife edge [24], slit [25], line source [26], or point source, and thus they require precision mechanical devices to scan these systems. In contrast, global methods eval- uate the MTF of the whole FPA. They consist in projecting a periodic pattern with known spatial frequencies onto the FPA. We can quote the generalized line spread function (GLSF) method [27] and the canted periodic target test (CPTT) [28]. In these two methods, a one-dimensional object target of period $d$ is projected onto the FPA.

The GLSF method is illustrated in Fig. 5. If the value of $d$ is different from a multiple of the sampling pitch $p_{s}$ of the FPA, the discrete orders of the MTF beyond the Nyquist frequency can be extracted, because they are folded on spatial frequencies of null amplitude. The CPTT is an extension of the GLSF method; it is illustrated in Fig. $\underline{6}$. It takes advantage of a tilt angle introduced between the axis of the onedimensional object target and the FPA columns to extract spatial frequencies beyond the Nyquist frequency. The advantage of the CPTT is that it does not require any condition on the period $d$ of the target with respect to the sampling pitch of the FPA. Thanks to the tilt angle introduced between the target and the detector axes, the frequencies beyond the Nyquist are folded along a line that is parallel to the sub-Nyquist part of the spectrum, but which does not overlap it if the tilt angle is different from $0^{\circ}$ and from a multiple of $45^{\circ}$.

\section{B. Description of the Method Proposed to Measure the MTF of a Multichannel System}

Based on previous work on MTF measurement, we propose a simple method to extract the MTF of a multichannel optical system based on the TOMBO principle. This method requires only a point source and no scanning system, leading to the great simplification of

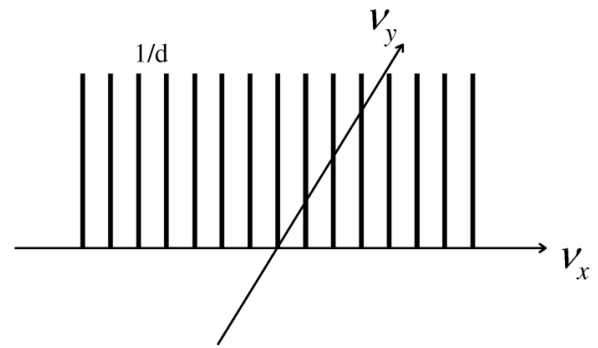

(a)
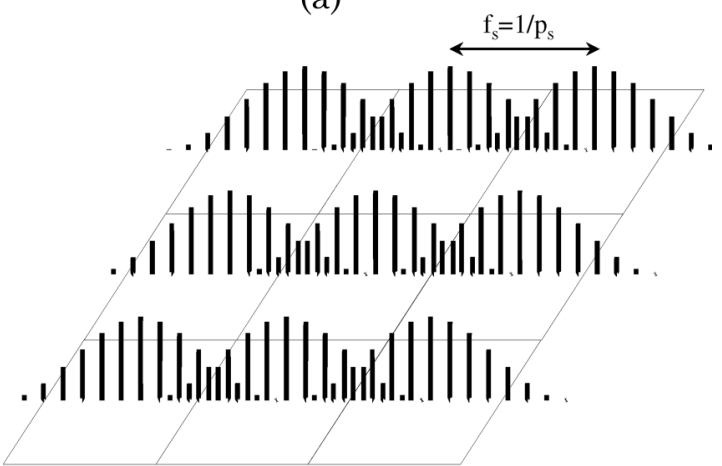

(b)

Fig. 5. GLSF method: (a) object target frequency spectrum, (b) effects of filtering by the pixel transfer function and sampling on the input spatial-frequency spectrum. 


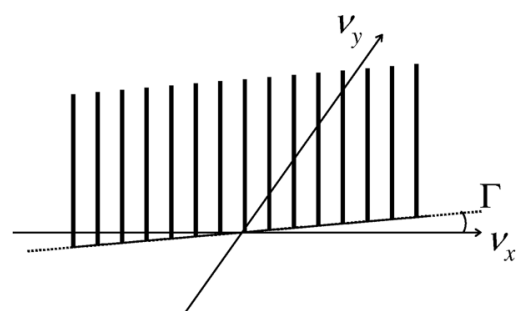

(a)

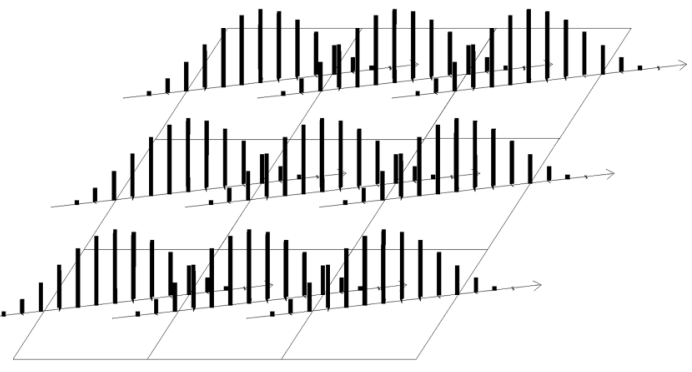

(b)

Fig. 6. CPTT: (a) object target frequency spectrum, (b) effects of filtering by the pixel transfer function and sampling on the input spatial-frequency spectrum.

the laboratory requirements and procedures. It takes advantage of the multiple channels to generate a twodimensional periodic pattern by projecting a point source.

The experimental PSF of a multichannel system based on the TOMBO principle is obtained by imaging a point source that is not resolved by the channels of the system. Then, an array of bright spots, described by Eq. (6), is formed in the focal plane of the multichannel system.

This array of bright spots can be seen as a twodimensional periodic pattern, in reference to the one-dimensional pattern used in the GLSF method. The experimental MTF of the multichannel optical system is obtained by calculating the modulus of the Fourier transform of the PSF, which leads to a two-dimensional structure of spatial frequencies of period $1 / d$ in the two directions, described by Eq. (7).

Therefore, according to the GLSF method, $d$ has to be different from a multiple of the sampling pitch $p_{s}$ of the FPA to extract frequencies beyond the Nyquist frequency. We can notice that this condition results in nonredundancy between the subimages.

However, in some cases, the period $d$ of the optical channels may be equal to the sampling pitch $p_{s}$ of the FPA. Therefore, the subimages are redundant, and the multichannel system is not well suited to retrieve frequencies greater than the Nyquist frequency in the final image. However, we propose a method to recover the nonredundancy; it is inspired by the CPTT and does not require any condition on the value of $d$. It consists in tilting the optical system in relation to the axes of the FPA. Nevertheless, as the MTF of a multichannel system is a two-dimensional structure, great care must be taken so that high spatial frequencies beyond the Nyquist frequency fold between low spatial frequencies and not on them.

\section{Condition on the Value of the Tilt Angle}

A schematic representation of the Fourier domain is given in Fig. 7. A frame delimiting the Nyquist frequency $1 /\left(2 \overline{p_{s}}\right)$ is illustrated. Replications of the tilted MTF appear around each multiple of the sampling frequency $1 / p_{s}$. The discrete structures generated by two of these replications are drawn. From this illustration, two equations can be extracted. The situation in which both equations are fulfilled corresponds to the case where the two discrete structures overlap:

$$
\cos \varphi=n \frac{p_{s}}{d}, \quad \sin \varphi=m \frac{p_{s}}{d},
$$

where $n$ and $m$ are two integers.

Each equation independently corresponds to the overlap of the structures in each tilted direction of the Fourier domain.

By summing the squares of the two equations, we obtain the following one:

$$
n^{2}+m^{2}=k^{2}=\left(\frac{d}{p_{s}}\right)^{2} .
$$

We can see that if $d / p_{s}$ is not an integer, Eq. (9) is never fulfilled, which means that there is no overlap between the structures, and the nonredundancy is obtained without tilting the optical system.

If $d$ is a multiple of $p_{s}\left(d=k p_{s}\right.$, where $k$ is an integer depending on the design of the optical system), Eqs. (8) become

$$
\cos \varphi=\frac{n}{k}, \quad \sin \varphi=\frac{m}{k} .
$$

If Eqs. (10) are fulfilled, some frequencies higher than the Nyquist frequency can be folded on low frequencies, and the method cannot be performed. Therefore, Eqs. (10) give the forbidden values of the tilt angle of the multichannel system.

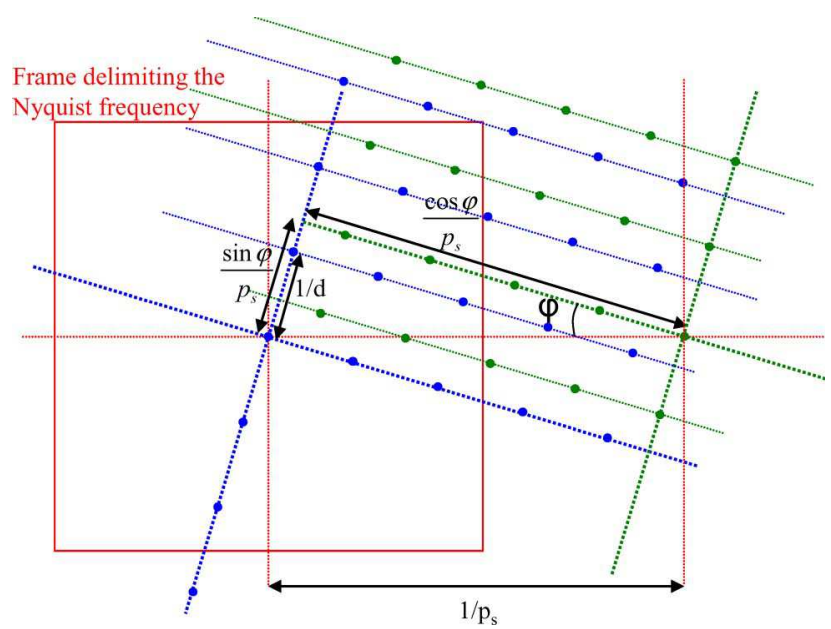

Fig. 7. (Color online) Two replications of the MTF of the multichannel system in the Fourier domain. The distances that enable us to find a condition on the value of the tilt angle are indicated. 
To solve Eqs. (10), we will first look for some integer pairs $(n, m)$ that fulfil these equations; then, we will deduce the value of the corresponding tilt angle $\varphi$. We will only consider values of $\varphi$ varying from $0^{\circ}$ to $45^{\circ}$, which will be justified below. We first begin by the study of particular integer pairs $(n, m)$.

If $(n, m)=(0,0)$, no value of $\varphi$ fulfils $\cos \varphi=$ $\sin \varphi=0$.

If $n=0$ and $m \neq 0, \cos \varphi=0$ is obtained for $\varphi=90^{\circ} . \sin \varphi=1$ leads to $m=k=d / p_{s}$, and $\varphi=$ $90^{\circ}$ is a forbidden value.

If $n \neq 0$ and $m=0, \sin \varphi=0$ is obtained for $\varphi=0^{\circ}$. $\cos \varphi=1$ leads to $n=k=d / p_{s}$, and $\varphi=0^{\circ}$ is a forbidden value. Indeed, in this case, the optical system is not tilted.

If $n \neq 0$ and $m \neq 0$, the combination of the two conditions of Eqs. (10) leads to the following equation:

$$
\tan \varphi=\frac{m}{n}
$$

The values of the integers $m$ and $n$ are given by Eq. (9), and the forbidden values of the tilt angle $\varphi$ are calculated with Eq. (11). Equation (9) consists in determining whether some points on the circle of radius $k$ have integer coordinates; it can be solved numerically for a given value of $k$ depending on the design of the multichannel system. This is illustrated in Fig. 8. We can see from this figure that if we determine an angle $\varphi$ between $0^{\circ}$ and $45^{\circ}$, which corresponds to an integer pair $(n, m)$, we can deduce all other forbidden angles. The integer pair related to the integer pair $(n, m)$ and which defines a tilt angle between $45^{\circ}$ and $90^{\circ}$ is $(m, n)$. Other integer pairs related to the integer pair $(n, m)$ and corresponding to values of the tilt angle between $90^{\circ}$ and $360^{\circ}$ are $(-n, m),(-n,-m),(n,-m)$. These integer pairs are related to other integer pairs, which are, respectively, $(-m, n),(-m,-n),(m,-n)$. All of these integer pairs are illustrated in Fig. 8 . We can notice that, unlike the CPTT and because two conditions have to be simultaneously fulfilled, the value of the tilt angle $\varphi=$ $45^{\circ}$ is not forbidden. In the following subsection, we will focus on the fundamental integer pair $(n, m)$ and the associated tilt angle between $0^{\circ}$ and $45^{\circ}$, knowing that all the other related integer pairs can be deduced from this fundamental integer pair.

Mathematically, $n$ and $m$ can vary from 0 to $k$. In practical cases, the maximum values for $n$ and $m$ are limited by the cutoff frequency of a single optical channel. Indeed, the highest order that can be contained in the MTF of a single channel is equal to the closest integer inferior to the product $\nu_{c} d$, where $\nu_{c}$ is the cutoff frequency of a single channel.

D. Interpretation of the Tilt Angle in Terms of Shift between the Subimages

In this subsection, we show that the tilt angle $\varphi$ between the optical system and the axes of the detector introduces a subpixel shift between the subimages. Figure $\underline{9}$ illustrates three adjacent bright spots of

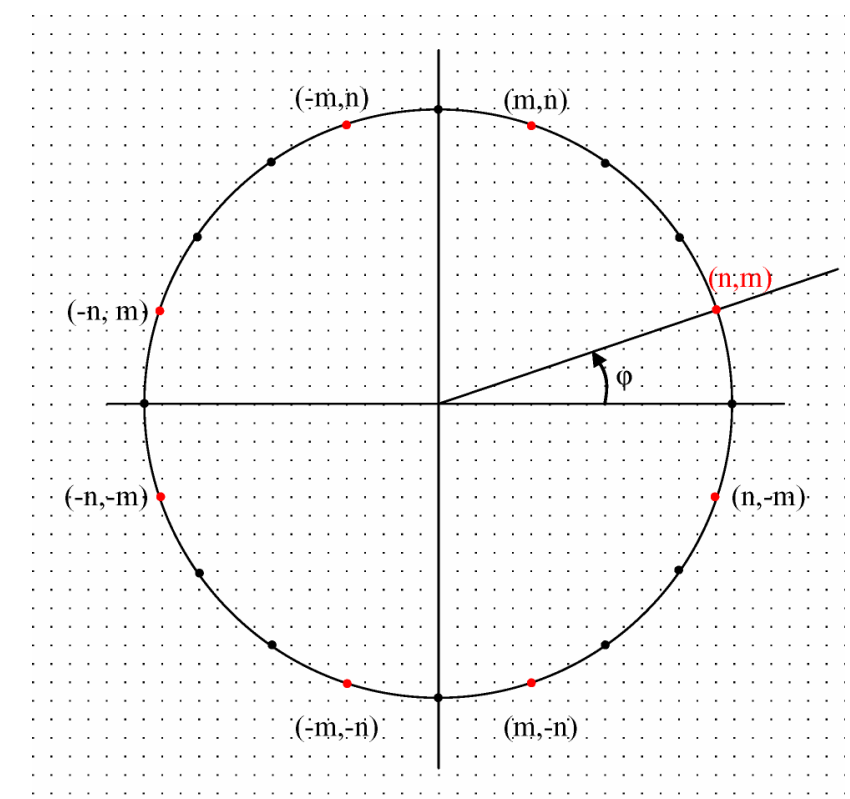

Fig. 8. (Color online) Integer pairs that can be deduced from an integer pair $(n, m)$ corresponding to a tilt angle $\varphi$ between $0^{\circ}$ and $45^{\circ}$.

the PSF. The shifts between two spots, and thus between adjacent channels, in the $x$ direction are given by $\Delta x_{1}$ and $\Delta y_{1}$. In the $y$ direction, the shifts are $\Delta x_{2}$ and $\Delta y_{2}$. They are expressed as follows:

$$
\begin{aligned}
& \Delta x_{1}=\Delta y_{2}=d \cos \varphi, \\
& \Delta y_{1}=\Delta x_{2}=d \sin \varphi .
\end{aligned}
$$

In order to introduce subpixel shifts between the subimages, $\Delta x_{1}$ and $\Delta y_{1}$ have to be different from a multiple of the sampling pitch $p_{s}$ of the FPA. In this case, we find the conditions expressed in Eq. (10).

\section{E. Optimal Value of the Tilt Angle}

We can see from Eq. (7) that, because of the finite dimensions of the detector, the bright spots of the MTF are extended in the Fourier domain. To extract

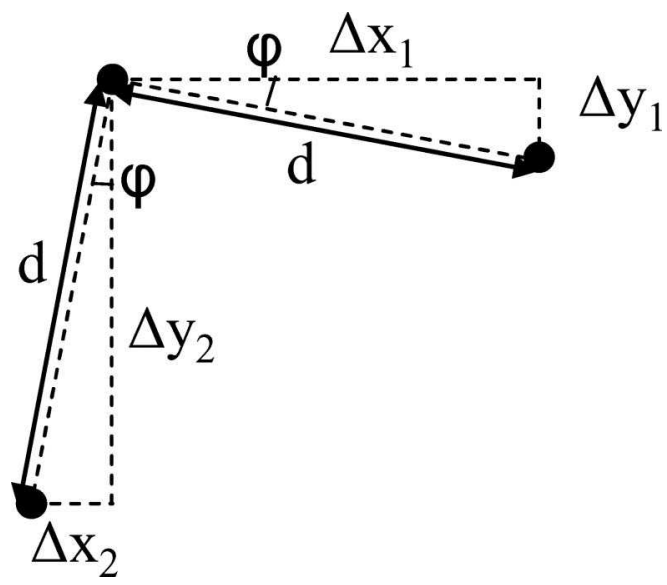

Fig. 9. Shifts between three adjacent optical channels. 
frequencies higher than the Nyquist frequency, a bright spot corresponding to one of these high frequencies has to be folded not too close to a bright spot corresponding to a spatial frequency less than the Nyquist frequency. The optimal position for a bright spot corresponding to a high frequency is to be placed at the center of the square formed by four lowfrequency bright spots, as illustrated in Fig. 10(a), or at least at half the distance between two adjacent bright spots in one direction if the value of the tilt angle is low [see Fig. 10(b)].

\section{Experimental Measurement of the MTF of a Multichannel Imaging System}

\section{A. Design of a Multichannel Imaging System}

We have designed a miniature multichannel camera called MULTICAM, working in the $[3 \mu \mathrm{m} ; 5 \mu \mathrm{m}]$ spectral bandwidth [21]. All the optics are integrated directly in the Dewar used to cool the detector, leading to a compact structure. The architecture of MULTICAM is made of multiple identical telescopes with parallel optical axes, and thus each channel images the same scene. It is composed of three stages of microlens arrays. The first microlens array focuses light in an intermediate image plane, where an array of field microlenses is placed. This latter corrects the

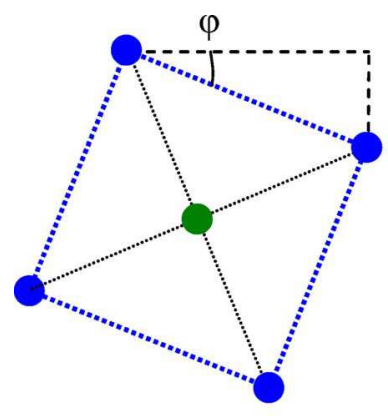

(a)

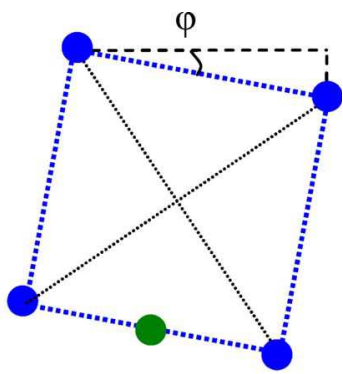

(b)
Fig. 10. (Color online) (a) Optimal value of the tilt angle, for which a bright spot corresponding to a high spatial frequency is at the center of the square formed by four low-frequency bright spots. (b) Situation in which a bright spot corresponding to a high spatial frequency is at half the distance between two lowfrequency bright spots.

field curvature aberration. The pinhole array placed on these microlenses delimits the FOV of each optical channel, which is equal to $6^{\circ} \times 6^{\circ}$, and avoids crosstalk between adjacent optical channels. The third stage of microlenses relays the image formed in the intermediate image plane on the detector plane. The $f$-number of a single channel is $\#=8.6$. The MULTICAM camera is associated with a focal plane

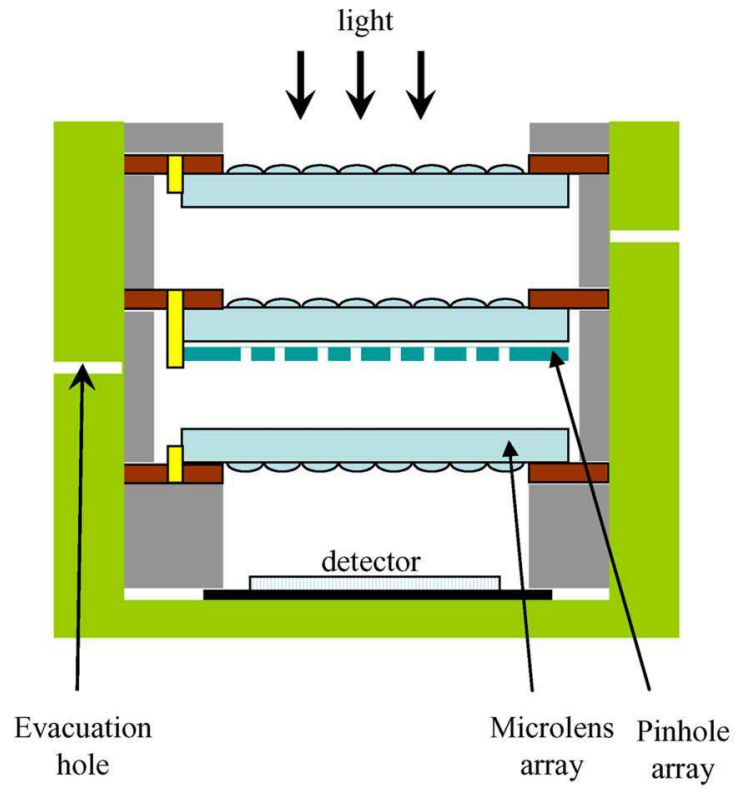

(a)

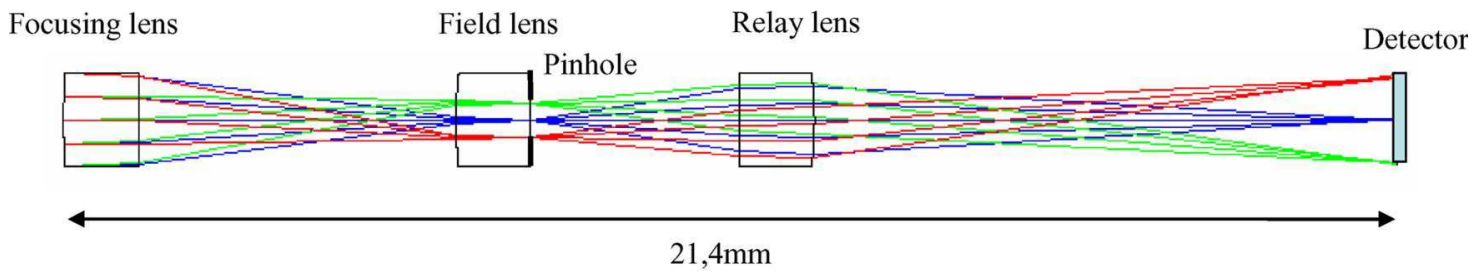

(b)

Fig. 11. (Color online) (a) Mechanical scheme of MULTICAM. (b) Optical scheme of one optical channel. 


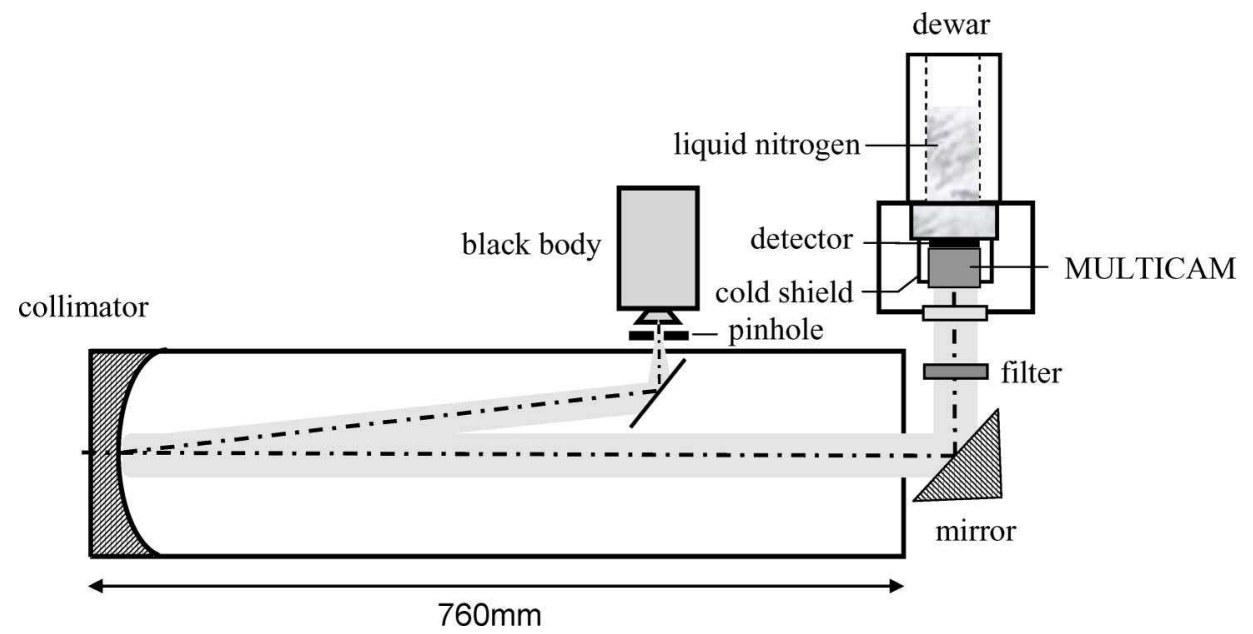

Fig. 12. Experimental setup for the measurement of the PSF of MULTICAM.

array with pixel pitch equal to $30 \mu \mathrm{m}$. Its architecture is illustrated in Fig. 11.

The period of the microlens arrays of MULTICAM is equal to $d=1.5 \mathrm{~mm}$; thus, it is a multiple of the sampling pitch of the detector $\left(p_{s}=30 \mu \mathrm{m}\right): d=k p_{s}$, with $k=50$. Following the method described in Section 3, we propose to obtain nonredundancy between the subimages by willingly tilting the optical system in relation to the axes of the detector.

\section{B. Calculation of the Forbidden Values of the Tilt Angle}

We solve numerically Eq. (9) for $k=50$. For $n$ and $m$ taking their values between 0 and $k$, two integer pairs $(n, m)$ corresponding to values of the tilt angle between $0^{\circ}$ and $45^{\circ}$ fulfil the condition expressed by

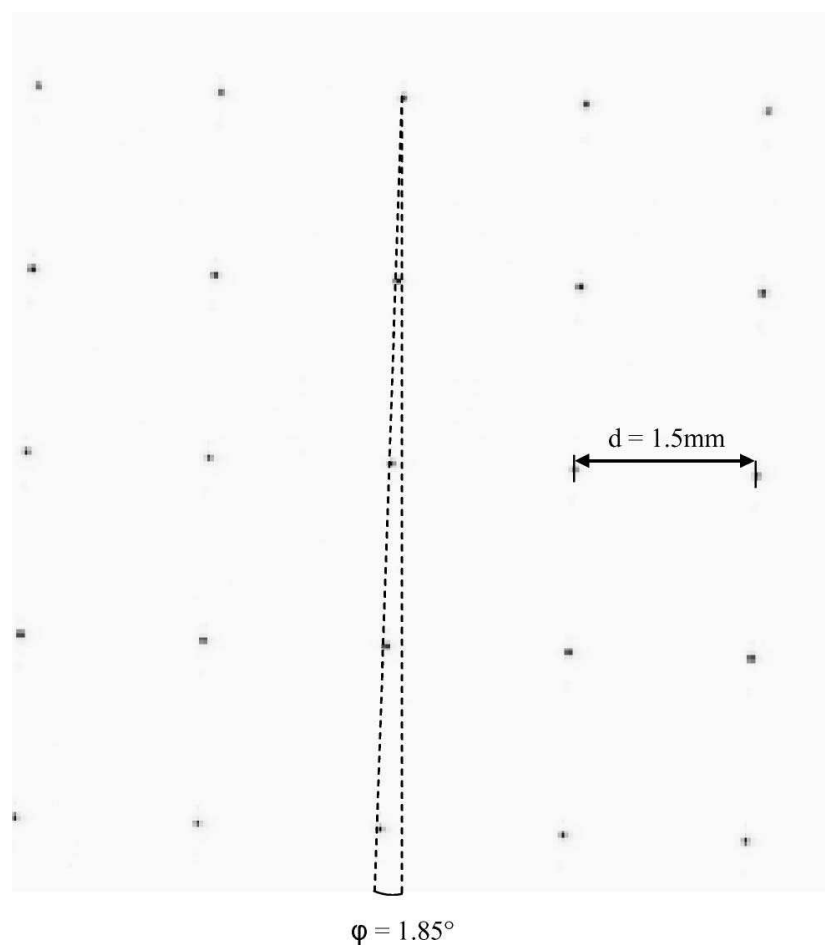

Fig. 13. Image of a nonresolved point source acquired by MULTICAM. The image is presented in negative contrast.
Eq. (9): $(n=48, m=14)$ and $(n=40, m=30)$. The forbidden values of the tilt angle are calculated from Eq. (11). They correspond, respectively, to the tilt angles $\overline{\varphi_{1}}=16.26^{\circ}$ and $\varphi_{2}=36.87^{\circ}$.

In practical cases, as we said in Subsection 3.C, the maximum values for $n$ and $m$ are limited by the cutoff frequency of a single channel of the optical system, which is given by $\nu_{c}=1 /\left(\lambda_{\text {mean }} \#\right)$, where $\lambda_{\text {mean }}$ is the mean wavelength of the spectral bandwidth. In the bandwidth $[3 \mu \mathrm{m} ; 5 \mu \mathrm{m}]$, we take $\lambda_{\text {mean }}=4 \mu \mathrm{m}$, and thus, $\nu_{c}=29 \mathrm{~mm}^{-1}$. Therefore, the maximum value for $n$ and $m$ is 43 . Thus, no overlap will result from the tilt angle $\varphi_{1}$ corresponding to $n=48$ and $m=14$.
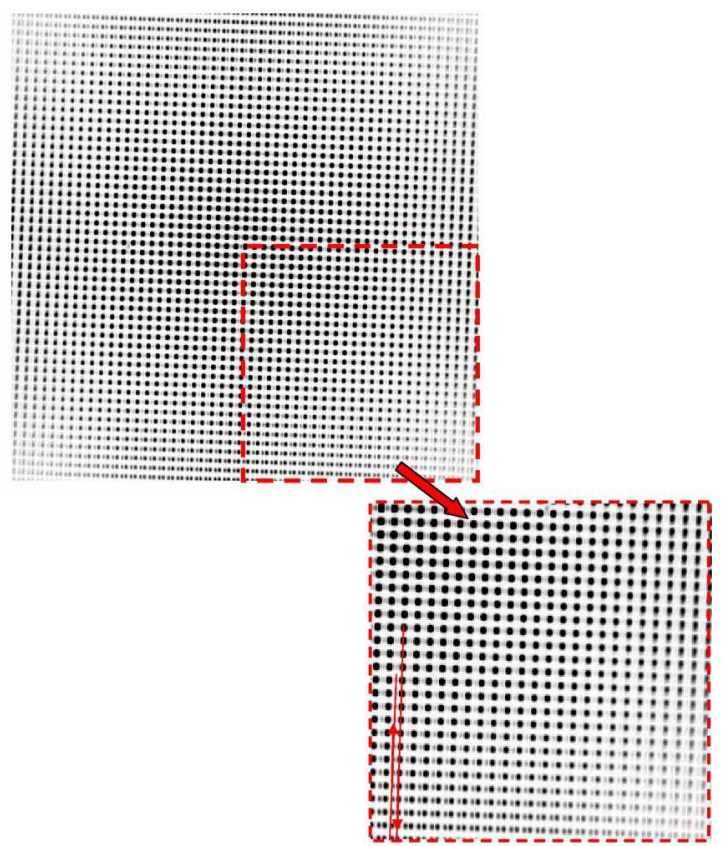

Fig. 14. (Color online) MTF of MULTICAM. Inset, spatial frequencies greater than the Nyquist frequency; the arrow pointing towards the bottom of the inset shows frequencies lower than the Nyquist frequency; the arrow pointing towards the top of the inset shows the folded frequencies greater than the Nyquist frequency. The image is presented in negative contrast. 
We are now looking for an optimal value for the tilt angle of the optical system. According to Subsection 3.E, if we choose to tilt the optical system with a slight angle $\varphi$, the bright spots corresponding to high spatial frequencies have to be at half the distance between two low-frequency adjacent bright spots. This situation is described by the following equation: $\sin \varphi=(q+1 / 2) / k$, where $q$ is an integer. Thus, the first optimal tilt angles are $\varphi=0.57^{\circ}$ for $q=0$, $\varphi=1.72^{\circ}$ for $q=1$, and $\varphi=2.86^{\circ}$ for $q=2$.

The MULTICAM camera has a tilt angle close to one of the optimal values: in practice, the tilt angle is equal to $\varphi=1.85^{\circ}$. According to the notation of Subsection 3.D, we calculate the shifts between the subimages as $\Delta x_{1}=\Delta y_{2}=1.499 \mathrm{~mm}$ and $\Delta y_{1}=$ $\Delta x_{2}=48 \mu \mathrm{m}$. Therefore, $\Delta x_{1}=\Delta y_{2} \simeq d$ and $\Delta y_{1}=$ $\Delta x_{2}=p_{s}+\Delta y_{\text {subpixel }}$, with $\Delta y_{\text {subpixel }}=18 \mu \mathrm{m}$. Thus, the tilt angle introduces a subpixel shift in the $y(x)$ direction for adjacent channels following the $x(y)$ direction.

\section{Measurement of the PSF of MULTICAM}

Using the camera MULTICAM, we acquire the image of a nonresolved point source. To generate the point source, we use a pinhole illuminated by a black body and placed at the focal point of a collimator with focal length $760 \mathrm{~mm}$. The test bench is illustrated in Fig. 12.

As the optical system is tilted in relation to the axes of the detector, the periodic structure of bright spots is also tilted with a tilt angle equal to $\varphi=1.85^{\circ}$. The image of a point source acquired by MULTICAM is illustrated in Fig. 13.

\section{Extraction of the MTF of MULTICAM}

The experimental MTF of the MULTICAM camera is obtained by calculating the Fourier transform of the PSF, which leads to a two-dimensional structure of spatial frequencies, illustrated in Fig. 14.

The experimental global MTF of MULTICAM extracted with this method is illustrated in Fig. 15: the MTF is measured for frequencies beyond the $\overline{\mathrm{Ny}}$ quist frequency. A section of the MTF along one direction is given in Fig. 16: by comparing the theoretical MTF to the experimental one, we can check that the MULTICAM camera is limited by the diffraction.

E. Illustration of the Resolution Enhancement of the Final Image by Superresolution Processing

The measurement of frequencies higher than the Nyquist frequency in the MTF shows that we can expect a resolution enhancement of the final image by applying a superresolution algorithm.

To show the resolution enhancement, we have acquired the image of a target whose frequency is undersampled by the focal plane array. This means that the spatial frequency of the target in the plane of the detector is higher than the Nyquist frequency of this latter. This target is obtained by illuminating a metallic target, illustrated in Fig. 17(a), with a black body. The image of the target acquired by MULTICAM is given

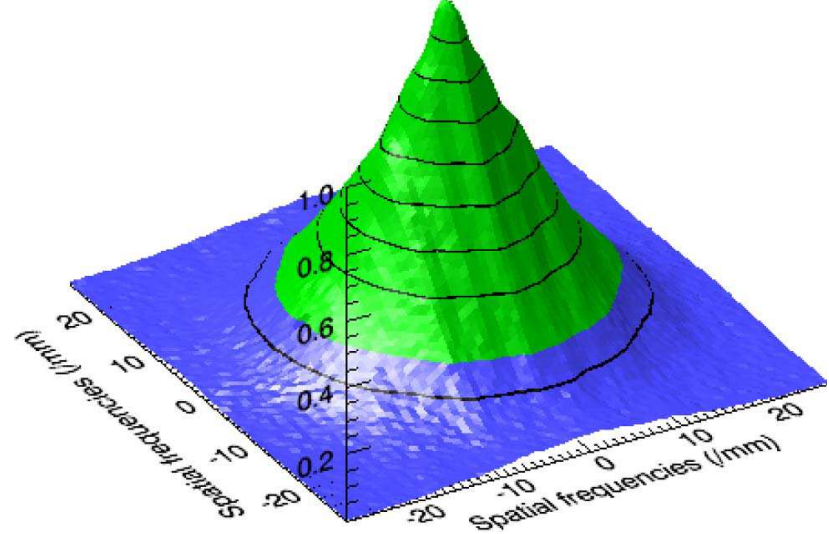

Fig. 15. (Color online) Experimental global MTF of MULTICAM. The green (upper) part of the plot is a representation of the MTF for frequencies lower than the Nyquist frequency. The blue (lower) part of the plot is a representation of the MTF for frequencies greater than the Nyquist frequency. Some level planes are indicated by the solid black curves.

in Fig. 17(b). We can check that the subimages are undersampled, because the period and the orientation of the slits in the image are different from the characteristics of the object target [see Fig. 17(c)]. In the Fourier domain, illustrated in the inset of Fig. 17(c) up to the Nyquist frequency, we can see that the spatial frequency of the target, which is greater than the $\mathrm{Ny}$ quist frequency, is folded on low spatial frequencies contained in the image.

To retrieve the high frequency of the target, we have developed an algorithm based on the classic shiftand-add technique $[29,30]$. It first evaluates and corrects the shift between the subimages. Then, the subimages are added so that subpixel information

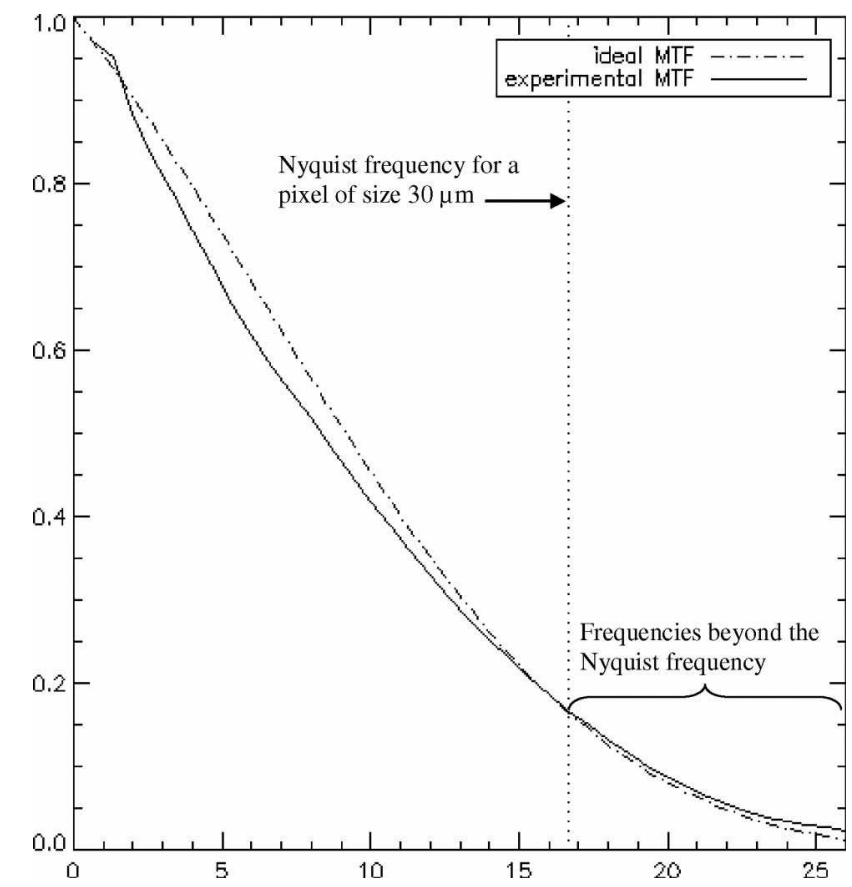

Fig. 16. Section of the MTF measured experimentally and compared with the theoretical MTF. 


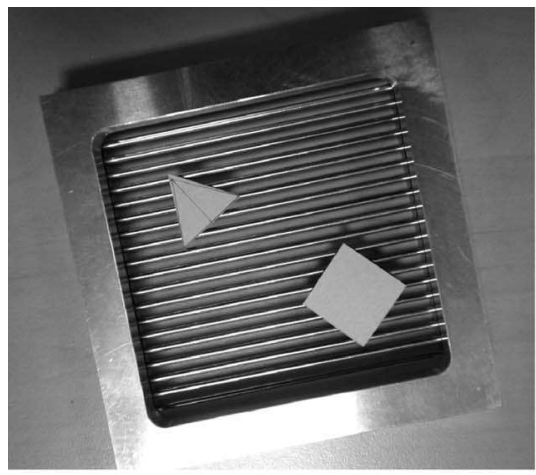

(a)

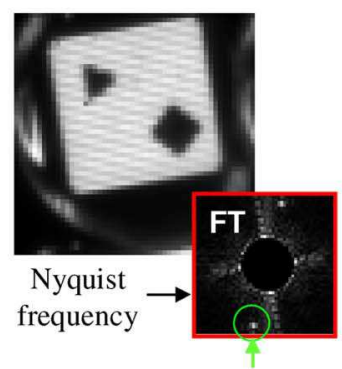

Spatial frequency of the target (folded on low frequencies)

(c)

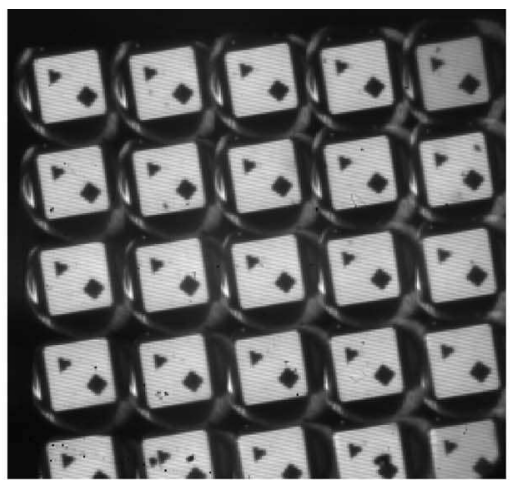

(b)

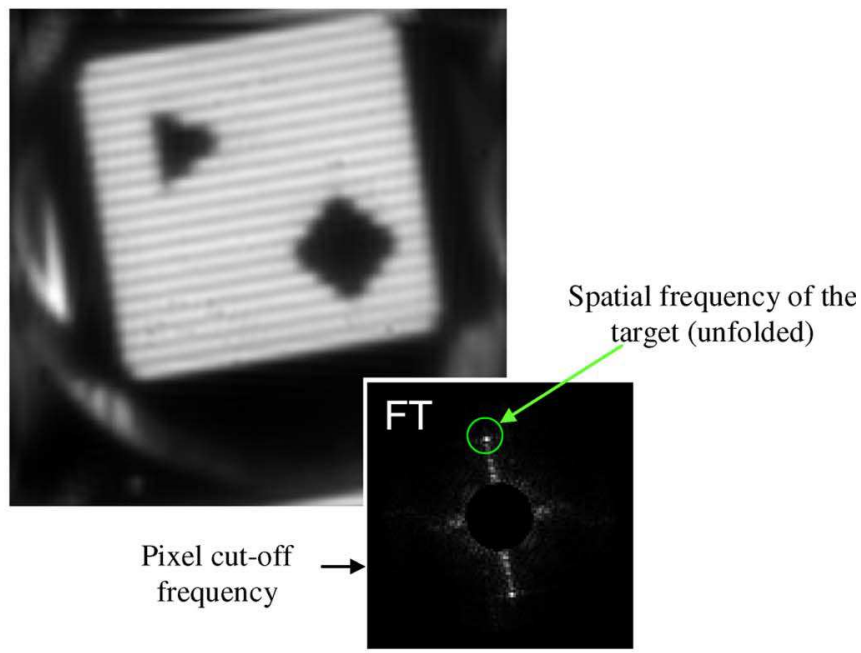

(d)

Fig. 17. (Color online) (a) Metallic target used as the object. (b) Image of the target acquired by MULTICAM. (c) One of the frames acquired by MULTICAM. Inset, Fourier transform of the image, illustrated below the Nyquist frequency. (d) Superresolved image. Inset, Fourier transform of the image, illustrated below the pixel cutoff frequency.

becomes available, leading to the retrieval of high spatial frequencies. Lastly, a Wiener filter deconvolution eliminates the blur caused by the finite size of pixels (deconvolution by the pixel transfer function) and thus enhances the contrast of the image.

The final image is illustrated in Fig. 17(d). Its Fourier transform illustrated until the pixel cutoff frequency (twice the Nyquist frequency) shows that the spatial frequency of the target is retrieved. We can see that the final image has the same characteristics as the object in terms of period and orientation of the slits.

\section{Conclusion}

In this paper, we have recalled the principles for the design of miniaturized optical systems leading to multichannel architectures based on the TOMBO concept. We have shown that multichannel systems combine the advantages of both a scanning monodetector, which enables us to sample the scene with a fine pitch, and a FPA, which provides the image of the scene in a single acquisition. These observations have lead to defining the global MTF of a multichannel optical system. Then, we have proposed an origi- nal method to measure the MTF of a multichannel imaging system, which only requires illuminating the optical system with a nonresolved point source. By taking advantage of the nonredundancy of the subimages (which can be obtained by introducing a tilt angle between the optical system and the axes of the detector), frequencies higher than the Nyquist frequency can be measured. Beyond the scope of evaluating the optical quality of a multichannel system, this method gives the performance of the multichannel system in terms of possible enhancement of the number of resolved points in the final image. Indeed, the measurement of frequencies greater than the Nyquist frequency suggests that a final image with an enhanced resolution can be built from the set of undersampled images provided by this optical system. Experimental data have demonstrated that a simple shift-and-add algorithm can unfold high frequencies in the final image, to enhance its resolution and contrast.

This work was sponsored by the Délégation Générale pour l'Armement (DGA) of the French Ministry of Defense. 


\section{References}

1. A. W. Lohmann, "Scaling laws for lens systems," Appl. Opt. 28, 4996-4998 (1989).

2. R. Völkel, M. Eisner, and K. J. Weible, "Miniaturized imaging systems," Microelectron. Eng. 67-68, 461-472 (2003).

3. J. Duparré, P. Dannberg, P. Schreiber, A. Bräuer, and A. Tünnermann, "Artificial apposition compound eye fabricated by micro-optics technology," Appl. Opt. 43, 4303-4310 (2004).

4. J. Duparré, P. Dannberg, P. Schreiber, A. Bräuer, and A. Tünnermann, "Thin compound-eye camera," Appl. Opt. 44, 2949-2956 (2005).

5. J. Duparré, P. Schreiber, A. Matthes, E. Pshenay-Severin, A. Bräuer, and A. Tünnermann, "Microoptical telescope compound eye," Opt. Express 13, 889-903 (2005).

6. G. Druart, N. Guérineau, R. Haïdar, S. Thétas, J. Taboury, S. Rommeluère, J. Primot, and M. Fendler, "Demonstration of an infrared microcamera inspired by Xenos peckii vision," Appl. Opt. 48, 3368-3374 (2009).

7. L. C. Laycock and V. A. Handerek, "Multi-aperture imaging device for airborne platforms," Proc. SPIE 6737, 673709 (2007).

8. J. Tanida, T. Kumagai, K. Yamada, S. Miyatake, K. Ishida, T. Morimoto, N. Kondou, D. Miyazaki, and Y. Ichioka, "Thin observation module by bound optics (TOMBO): concept and experimental verification," Appl. Opt. 40, 1806-1813 (2001).

9. M. Shankar, R. Willett, N. Pitsianis, T. Schulz, R. Gibbons, R. Te Kolste, J. Carriere, C. Chen, D. Prather, and D. Brady, "Thin infrared imaging systems through multichannel sampling," Appl. Opt. 47, B1-B10 (2008).

10. R. Shogenji, Y. Kitamura, K. Yamada, S. Miyatake, and J. Tanida, "Multispectral imaging using compact compound optics," Opt. Express 12, 1643-1655 (2004).

11. K. Nitta, R. Shogenji, S. Miyatake, and J. Tanida, "Image reconstruction for thin observation module by bound optics by using the iterative backprojection method," Appl. Opt. 45, 2893-2900 (2006).

12. Y. Kitamura, R. Shogenji, K. Yamada, S. Miyatake, M. Miyamoto, T. Morimoto, Y. Masaki, N. Kondou, D. Miyazaki, J. Tanida, and Y. Ichioka, "Reconstruction of a high-resolution image on a compound-eye image-capturing system," Appl. Opt. 43, 1719-1727 (2004).

13. K. Choi and T. J. Schulz, "Signal-processing approaches for image-resolution restoration for TOMBO imagery," Appl. Opt. 47, B104-B116 (2008).

14. R. Barnard, V. P. Pauca, T. C. Torgersen, R. J. Plemmons, S. Prasad, J. van der Gracht, J. Nagy, J. Chung, G. Behrmann, S. Mathews, and M. Mirotznik, "High-resolution iris image reconstruction from low-resolution imagery," Proc. SPIE 6313, D1-D13 (2006).
15. J. Fanning, J. Miller, J. Park, G. Tener, J. Reynolds, P. O'Shea, C. Halford, and R. Driggers, "IR system field performance with superresolution,” Proc. SPIE 6543, 65430Z (2007).

16. M. D. Robinson and D. G. Stork, "Joint digital-optical design of superresolution multiframe imaging systems,” Appl. Opt. 47, B11-B20 (2008).

17. K. Krapels, R. G. Driggers, E. Jacobs, S. Burks, and S. Young, "Characteristics of infrared imaging systems that benefit from superresolution reconstruction," Appl. Opt. 46, 4594-4603 (2007).

18. M. W. Haney, "Performance scaling in flat imagers," Appl. Opt. 45, 2901-2910 (2006).

19. F. Champagnat, G. Le Besnerais, and C. Kulcsàr, "Statistical performance modeling for superresolution: a discrete datacontinuous reconstruction framework," J. Opt. Soc. Am. A 26, 1730-1746 (2009).

20. A. V. Kanaev, J. R. Ackerman, E. F. Fleet, and D. A. Scribner, "TOMBO sensor with scene-independent superresolution processing," Opt. Lett. 32, 2855-2857 (2007).

21. G. Druart, N. Guérineau, R. Haïdar, M. Tauvy, S. Thétas, S. Rommeluère, J. Primot, J. Deschamps, and E. Lambert, "MULTICAM: a miniature cryogenic camera for infrared detection," Proc. SPIE 6992, 699215 (2008).

22. C.-L. Tisse, F. Guichard, and F. Cao, "Does resolution really increase image quality?" Proc. SPIE 6817, 68170Q (2008).

23. A. Papoulis, "Generalized sampling expansion," IEEE Trans. Circuits Syst. 24, 652-654 (1977).

24. S. E. Reichenbach, S. K. Park, and R. Rarayanswamy, "Characterizing digital image acquisition devices," Opt. Eng. 30, 170-177 (1991).

25. M. A. Chambliss, J. A. Dawson, and E. J. Borg, "Measuring the MTF of undersampled staring IRFPA sensors using 2D discrete Fourier transform," Proc. SPIE 2470, 312-324 (1995).

26. G. C. Holst, "Infrared imaging system testing," in Infrared and Electro-optical Systems Handbook, M. C. Dudzik, ed. (SPIE, 1993), Vol. 4, pp. 223-232.

27. M. Chambon, J. Primot, and M. Girard, "Modulation transfer function assessment for sampled imaging system: application of the generalized line spread function to a standard infrared camera," Infrared Phys. Technol. 37, 619-626 (1996).

28. N. Guérineau, J. Primot, M. Tauvy, and M. Caes, "Modulation transfer function measurement of an infrared focal plane array by use of the self-imaging property of a canted periodic target," Appl. Opt. 38, 631-637 (1999).

29. J. Shi, S. E. Reichenbach, and J. D. Howe, "Small-kernel superresolution methods for microscanning imaging systems," Appl. Opt. 45, 1203-1214 (2006).

30. M. Elad and Y. Hel-Or, "A fast super-resolution reconstruction algorithm for pure translationnal motion and common spaceinvariant blur," IEEE Trans. Image Process. 10, 1187-1193 (2001). 